\section{Thrombophilia and abdominal vessel thrombosis in a Greek University hospital: a five-year experience}

\author{
loannis Starakis, ${ }^{1}$ Elias Mazokopakis, ${ }^{2}$ \\ Athina Mougiou, 'Angelos Koutras, ${ }^{1}$ \\ Charalambos A. Gogos' \\ 'Department of Internal Medicine, Patras \\ University Hospital, Rion-Patras, Greece; \\ 2Department of Internal Medicine, Naval \\ Hospital of Crete, Chania, Greece
}

\section{Abstract}

Thrombophilia, either congenital or acquired, has foremost consequences in the abdominal vessels. We review here the cases of patients admitted with abdominal vessel thrombosis over a five-year period. Our data focused on gender and age at diagnosis, site of thrombosis, previous thromboembolic events, underlying conditions, and family history. Investigations included measurement of protein $\mathrm{C}$, protein $\mathrm{S}$, activated $\mathrm{PC}$ resistance, and antithrombin, and screening for factor $\mathrm{V}$ Leiden, prothrombin G20210A, the C677T variant of the methylenetetrahydrofolate reductase gene, and V617F JAK2 mutation, r lupus anticoagulant, antiphospholipid antibodies and paroxysmal nocturnal hemospherinouria, and also serum folate, vitamin 12 , and total homocysteine concentrations. Twenty-nine patients were admitted and 18 of their family members also underwent the same thrombophilia investigations. Eighteen patients (62.1\%) presented with portal vein thrombosis (PVT), five patients (17.2\%) with mesenteric vein thrombosis (MVT), four patients (13.8\%) with splenic vein thrombosis (SVT), and two (6.9\%) patients with hepatic vein thrombosis (HVT). There was a high incidence of congenital thrombophilia (37.9\%), acquired thrombophilic conditions (27.6\%), or both (20.7\%). Sixteen of 18 patients with PVT, four of five patients with MVT, all four patients with SVT, and one of two patients with HVT had one or more thrombophilic risk factors. In $13.8 \%$ of the patients no underlying condition was identified. We concluded that thrombophilia may have major implications in the pathogenesis of abdominal vessel thrombosis in adult life, and a thorough thrombophilia investigation should be performed in all these patients.

\section{Introduction}

A key hypothesis demarcating the pathogenetic mechanisms of venous thromboembolic events (VTE), frequently called Virchow's triad, ${ }^{1}$ suggests that VTE arises as a consequence of adjustments in blood flow (i.e. stasis), vascular endothelial injury, and congenital or acquired hypercoagulable states. Inherited thrombophilia is a genetic propensity to VTE that habitually affects younger patients ( $<50$ years of age) and frequently is recurring. The most common inherited hypercoagulable states are the factor V Leiden (FVL) and prothrombin gene (prothrombin G20210A [FII G20210A], the C677T variant of the methylenetetrahydrofolate reductase [MTHFR] gene, and V617F JAK2) mutations, which jointly are accountable for almost $50 \%$ of cases. Protein S (PRS), protein C (PRC), and antithrombin (AT) defects account for most of the remaining cases, while a rare cause is one of the various dysfibrinogenemias.

Acquired risk factors or predisposing conditions for thrombosis include hospital admission in the past three months or current hospitalization, recent surgery (especially orthopedic), infection in the past three months, presence of a central venous catheter, malignancy, trauma, immobilization, pregnancy, the use of oral contraceptives or hormone replacement therapy, myeloproliferative disorders (MPD), and antiphospholipid syndrome. Furthermore, other conditions influencing thromboembolic incidents encompass the administration of thalidomide, lenalidomide, tamoxifen, and bevacizumab, congestive heart failure, paroxysmal nocturnal hemospherinouria (PNH), nephrotic syndrome, inflammatory bowel disease, hyperviscosity syndromes such as Waldenstrom's macroglobulinemia and multiple myeloma, leukostasis in acute leukemia, sickle cell disease and AIDS. ${ }^{2}$ Irrespective of whether the patient has a congenital and/or an acquired risk factor for VTE, the occurrence of an affirmative history of VTE in the family recently has been demonstrated to represent a strong supplementary risk factor for VTE. ${ }^{3}$ Portal vein thrombosis (PVT), Budd-Chiari syndrome (BCS), and mesenteric vein thrombosis (MVT) have a diversity of clinical representations ranging from an entirely asymptomatic patient to a patient with acute abdominal pain. Moreover, some patients may present with chronic hepatomegaly and/or complications like portal gastropathy, esophageal varices, severe hypersplenism, ascites, and liver failure necessitating liver transplantation.

Thrombophilia, either congenital or acquired, has foremost consequences in the abdominal vessels. The management strategies for these complications of intra-abdominal vessel thrombosis are not different from
Correspondence: I. Starakis, Patras University Hospital, 26500 Rion-Patras, Greece.

E-mail: istarakis@yahoo.com

Key words: abdominal vessels, thrombosis, thrombophilia.

Contributions: All authors have made substantial contributions to conception and design, acquisition of data, and analysis and interpretation of data. The corresponding author has been involved in drafting the manuscript and revising it critically for important intellectual content. All authors have given final approval of the version to be published.

Conflict of interest: the authors report no conflicts of interest.

Acknowledgements: this study has been approved by the Ethics Committee of Patras University hospital.

Received for publication: 23 October 2009

Revision received: 26 December 2009

Accepted for publication: 27 December 2009

This work is licensed under a Creative Commons Attribution 3.0 License (by-nc 3.0)

(C) Copyright I. Starakis et al., 2010

Licensee PAGEPress, Italy

Gastroenterology Insights 2010; 2:e2

doi:10.4081/gi.2010.e2

their equivalent in cirrhotic portal hypertension, but the prognosis is unquestionably better in the former cases. Physicians must be vigilant and their efforts should be focused on an early diagnosis and the implementation of all appropriate therapeutic proceedings.

The aim of this study was to investigate all cases with abdominal vessel thrombosis admitted to our hospital over a five-year period thoroughly for thrombophilia (inherited and acquired). Our intention was to recognize the prevalence of congenital hypercoagulable states and also acquired risk factors or predisposing underlying conditions for thrombosis in this cohort of patients and their family members.

\section{Patients and Methods}

We reviewed the case records of all patients who had had abdominal vessel thrombosis at Patras University Hospital during a five-year period between 2001 and 2006. The diagnosis was confirmed by contrast angiography in most patients but in a minority of cases it was documented by non-invasive imaging such as computerized tomography scanning of the abdomen, color Doppler ultrasonography, 
and/or magnetic resonance angiography. Data collected included gender and age at diagnosis, underlying conditions, site of thrombosis and documentation methods, the occurrence of previous thromboembolic events, and related precipitating factors like surgery, sepsis, malignancy, chemotherapy, and family history. Investigations included measurement of PRC, PRS, activated PC resistance (APC-R), and AT, and screening for FVL, FII G20210A, MTHFR C677T, and V617F JAK2 mutations, $r$ lupus anticoagulant (LA), antiphospholipid antibodies (APA), and PNH, and also serum folate, vitamin B12, and total serum homocysteine (tHcy) concentrations.

Antigenic PRC and PRS levels were measured using human protein $\mathrm{C} / \mathrm{S}$ "NL" NANORID $^{\mathrm{TM}}$ radial immunodiffusion kit (the Binding Site Ltd, Birmingham, UK), and the diagnosis of PRC and PRS deficiencies was confirmed if antigenic PRC and PRS levels were less than $60 \%$ of normal. At this point it should be mentioned that the related test is highly variable still and not completely reliable. AT was measured by a chromogenic assay (Immuno Ltd, Austria). APC-R was measured by the Coatest assay (first generation assay, Chromogenix AB, Sweden). The detection of the FVL mutation was carried out using blood in a polymerase chain reaction (PCR), followed by Mnl I digestion of an amplified factor $\mathrm{V}$ DNA fragment. Detection of the prothrombin G20210A allele was carried out in the same manner using the Hind III restriction enzyme. LA testing included partial thromboplastin time (PTT), prothrombin time (PT), dilute Russell viper venom screen, and a hexagonal (II) phase phospholipid assay (Staclot-LA test). In addition, a thrombin time test and a fibrinogen test were done to rule out heparin contamination and abnormal deficiency in fibrinogen, respectively. APA were tested using an enzyme-linked immunosorbent assay (ELISA) immunological test, which screens for the presence of $\beta 2$-glycoprotein-1-dependent anticardiolipin antibodies. Plasma Hcy concentrations were measured by high performance liquid chromatographytandem mass spectrometry and concentrations $>15 \mu \mathrm{mol} / \mathrm{L}$ were regarded as hyperhomocystinemia. Genotyping for the MTHFR C677T polymorphisms was carried out by PCR followed by Hinfl enzyme digestion. The V617F JAK2 mutation was detected on genomic DNA extracted from peripheral granulocytes on an ABI7700 apparatus using real-time PCRbased mutation detection with allele specific Taqman probes and with a sensitivity of about $2 \%$. To establish the diagnosis of $\mathrm{PNH}$, the patient's erythrocytes and neutrophils were stained with directly conjugated antibodies specific for two of several glycophosphatidylinositol-linked proteins; for example, CD55, CD58, CD59, and CD66b. Then the stained cells were analyzed by flow cytometry. The percentage of the affected erythrocytes and neutrophils were reported as were the antigens, which were expressed at lower levels.

None of the patients had leukocytosis (median WBC count $\sim 170 \times 10^{9} / \mathrm{L}$ ) with a predominance of neutrophils in different stages of maturation and absolute basophilia, indicating chronic myelogenous leukemia. Increased platelet counts ( $\geq 600 \times 10 \% / L)$ were not noticed, indicating essential thrombocythemia (ET). Furthermore, none of our patients had increased hemoglobin ( $>18.5 \mathrm{~g} / \mathrm{dL}$ in men; $>16.5 \mathrm{~g} / \mathrm{dL}$ in women) and hematocrit, white blood cell (WBC) count $>12 \times 10^{9} / \mathrm{L}$ and platelet count $>400 \times 10 \% / L$, indicating polycythemia vera (PV). In addition, mild to marked thrombocytosis accompanied by mild to moderate leukocytosis and mild anemia, pointing to a prefibrotic stage of chronic idiopathic myelofibrosis, was not noticed; neither were moderate to marked anemia, leukoerythroblastosis or prominent red blood cell poikilocytosis with teardrop shapes, suggesting a fibrotic stage of chronic idiopathic myelofibrosis. There was no indication of chronic eosinophilic leukemia/hypereosinophilic syndrome (eosinophilia $[\geq 1.5 \times 10 \%$ ] and myeloblasts $<20 \%$ ), and none of the patients presented with the diagnostic criteria for chronic neutrophilic leukemia $\left(\mathrm{WBC}\right.$ count $\geq 25 \times 10^{9} / \mathrm{L}$, segmented neutrophils and bands $>80 \%$, immature granulocytes $<10 \%$, and myeloblasts $<1 \%$ ). The V617F ${ }^{\text {JAK2 }}$ kinase mutation, which recently was discovered to be associated with PV, ET, and chronic idiopathic myelofibrosis, was not detected in our patients. It is known that peripheral blood mutation screening cannot substitute for bone marrow histology at this time, but owing to lack of supportive evidence, submitting these patients to a bone marrow aspiration has not been considered as a necessary measure. Yet, the V617F JAK2 mutation is not detected always in patients with PV and is absent in almost half of patients with ET and idiopathic myelofibrosis. All these patients are regularly visiting our hospital still, and to date there has not been any indication from the clinical examination and/or the laboratory findings suggesting the development of a myeloproliferative disease.

\section{Results}

During the five-year study period, 29 patients were admitted with symptomatic abdominal vessel thrombosis and all underwent thrombophilia investigations. There were 13 males and 16 female patients. Their ages at diagnosis of the abdominal thrombotic event ranged from 18 to 64 years. None had been investigated for thrombophilia previously. Ten patients (34.5\%) had suffered another thrombotic incident in the past (six had deep vein thombosis, two had cerebrovascular accidents, one had pulmonary thromboembolism, and one had retinal vein thrombosis). The mean time between the first and second thrombotic event was 25 months (range: from five months to ten years). Of these ten patients, five had congenital thrombophilia. Of these, one had FVL and no other risk factors at the time of the first thrombotic event; one had FVL and a viral infection of the respiratory tract; one had PRS deficiency and an acute appendicitis; one had PRS and AT deficiencies, and acute cholecystitis with gram-negative sepsis; and one had FII G20210A and acute pyelonephritis with gramnegative bacteremia. In the five patients without congenital thrombophilia, acquired predisposing factors were identified in three: myeloproliferative disease in one and liver cirrhosis in two. All patients investigated had normal renal function and none of them was on anticoagulation therapy. Female patients were not receiving oral contraceptives, hormone replacement, or drugs interfering with Hcy metabolism. None of them was pregnant or in the postpartum period. Not all family members of each index case were investigated, mainly because of their unwillingness, and only 18 family members of eight patients underwent the same extensive thrombophilia investigation. Three out of these 18 patients' relatives had a history of thrombotic event and one of them was found to have FVL.

No combined thromboses were noticed in our patients. Eighteen patients (62.1\%) presented with PVT, five patients (17.2\%) with MVT, four patients (13.8\%) with splenic vein thrombosis (SVT), and two (6.9\%) patients with hepatic vein thrombosis (HVT) (Table 1). Twenty-five (86.2\%) of the 29 patients had one or more predisposing factors when presented with the abdominal thrombotic event. Eight patients (27.6\%) had only acquired factors, eleven (37.9\%) congenital factors alone, and six $(20.7 \%)$ had both. No identifiable predisposing factors were detected in four (13.8\%) patients. The most frequently observed acquired factor was liver cirrhosis, seen in six out of 29 patients (20.7\%); all presented with PVT. Five patients (17.2\%) had been diagnosed previously as suffering from MPD (two had ET, and three suffered from PV) (Table 2). Three patients (10.3\%) had genetic hemochromatosis, and one patient (3.4\%) had been operated on 30 days previously because of a suprasellar anaplastic cerebral oligodendroglioma.

In total, we found an underlying congenital thrombophilic condition in 17 (58.6\%) out of the 29 patients, and in six (20.7\%) more than one genetic thrombophilic factor were detected. The most common defect noticed was PRS deficiency, which was found in seven (24.1\%) of the 29 patients. PRS deficiency was also the most common (33.3\%) congenital underlying defect found in patients with PVT. Homo- 
zygosity for the MTHFR 677T allele associated with increased serum homocysteine levels and low folate values was found in five (17.2\%) patients. In fact, homozygosity for the C677T variant of MTHFR gene represented the only predisposing thrombophilic state in three (75\%) out of four patients with SVT, but in only one (5.5\%) out of 18 patients with PVT. A mutation in the gene encoding FVL was found in five (17.2\%) patients, with four affected patients heterozygous and one homozygous. Heterozygous FVL mutation was the only predisposing factor in one (5.5\%) out of 18 patients with PVT but it was detected in four (80\%) out of five patients with MVT. None of our patients had the V617F JAK2 mutation.

Five (17.2\%) patients had PRC deficiency, and this defect ranked second after PRS deficiency in patients with PVT (22.2\%). Four (13.8\%) patients had AT deficiency, which ranked third (16.6\%) after PRS and PRC deficiency in patients with PVT. The FII G20210A mutation was not detected in any of our patients, and APC-R was found in only one (3.4\%) patient suffering from MVT combined with another four genetic thrombophilic factors. Vitamin B12 plasma concentrations were normal in all studied patients. None of our patients had LA, APA, or PNH.

\section{Discussion}

\section{Portal vein thrombosis}

In total, 16 (88.8\%) of our PVT patients had one or more underlying prothrombotic risk factors in our series, and these results are comparable with those reported in other studies, ${ }^{47}$ emphasizing the high incidence rates of the causal prothrombotic conditions. Of our PVT patients, $38.9 \%, 22.2 \%$, 27.8\%, and $11 \%$ had only acquired, merely congenital, both acquired and congenital, and no thrombophilic conditions, respectively. Overall, the prevalence of PRS (33.3\%), PRC (22.2\%), and AT (16.6\%) deficiencies found in our PVT patients was compatible with the findings reported in other surveys. ${ }^{4.7}$ One (5.5\%) PVT patient was heterozygous for FVL, and in one (5.5\%) was MTHFR 677T allele homozygosity detected along with hyperhomocysteinemia and low

Table 1. Site of the thrombosis and underlying thombophilic conditions.

\begin{tabular}{llllll} 
Site & pts (\%) & $\begin{array}{l}\text { Acquired } \\
\text { pts }(1 \%)\end{array}$ & $\begin{array}{l}\text { Congenital } \\
\text { pts }(1 \%)\end{array}$ & $\begin{array}{l}\text { Both } \\
\text { pts }(1 \%)\end{array}$ & $\begin{array}{l}\text { None } \\
\text { pts }(/ \%)\end{array}$ \\
PVT & $18 / 29(62.1 \%)$ & $7 / 18(38.9 \%)$ & $4 / 18(22.2 \%)$ & $5 / 18(27.8 \%)$ & $2 / 18(11.1 \%)$ \\
MVT & $5 / 29(17.2 \%)$ & $0 / 5(0 \%)$ & $4 / 5(80 \%)$ & $0 / 5(0 \%)$ & $1 / 5(20 \%)$ \\
\hline SVT & $4 / 29(13.8 \%)$ & $1 / 4(25 \%)$ & $3 / 4(75 \%)$ & $0 / 4(0 \%)$ & $0 / 4(0 \%)$ \\
HVT & $2 / 29(6.9 \%)$ & $0 / 2(0 \%)$ & $0 / 2(0 \%)$ & $1 / 2(50 \%)$ & $1 / 2(50 \%)$ \\
\hline Total & $29(100 \%)$ & $8 / 29(27.6 \%)$ & $11 / 29(37.9 \%)$ & $6 / 29(20.7 \%)$ & $4 / 29(13.8 \%)$ \\
\hline
\end{tabular}

pts, patients; PVT, portal vein thrombosis; MVT, mesenteric vein thrombosis; SVT, splenic vein thrombosis; HVT, hepatic vein thrombosis. Acquired: liver cirrhosis, myeloproliferative diseases (polycythemia vera and essential thrombocytosis), genetic hemochromatosis, suprasellar anaplastic cerebral oligodendroglioma. Congenital: protein S (PRS), protein C (PRC) and antithrombin (AT) deficiencies, Factor V Leiden, homozygosity for the methylenetetrahydrofolate reductase C677T allele, activated PC resistance.

folate. This last case is of particular interest because there are only three cases reported in the literature to date. ${ }^{8}$

Although FVL is the most common hereditary thrombophilic defect in individuals of European origin, with an allele frequency ranging from $1.4 \%$ to $7 \%,{ }^{9}$ our results corroborate with previous data indicating its minor pathogenetic role in PVT. ${ }^{10,11}$ On the other hand, our study did not confirm either the increased frequency of prothrombin G20210A mutation in PVT or the suggestion that homozygous MTHFR C677T genotype is strongly associated with this medical entity. ${ }^{12-15}$ Furthermore, we could not substantiate the results of other reports ${ }^{16}$ concerning the role of LA in PVT, as LA was recovered neither in any PVT patient nor in any other of our patients. In our study, MPD as a cause of PVT ranked second (22.2\%) after liver cirrhosis (33.3\%), but most of the previous studies found them to prevail with prevalence rates ranging from $30 \%$ to $48 \%{ }^{4 \cdot 7}$ Idiopathic PVT has been associated with latent MPD that becomes apparent only years after the diagnosis of PVT. Our patients (three with PV and one with ET) had been suffering from MPD for years. The occurrence of PVT during the course of all MPD is not rare (7.1\%) and the highest incidence of thrombosis has been reported in the PV group. ${ }^{17}$

\section{Portal vein thrombosis patients and liver cirrhosis}

All six cirrhotic patients in our study presented with PVT and probably should have been analyzed separately, as thrombophilic factors are quite different in patients with and without liver cirrhosis. Two out of these six cirrhotic patients had no other thrombophilic condition, and in the other four the cirrhosis was accompanied by PRC, PRS, and AT deficiencies. Because of reduced liver synthetic activity, cirrhotic patients often have low levels of PRC, PRS, and AT, as well as of coagulation factors. This makes it difficult to distinguish congenital from acquired deficiencies of these proteins in cirrhotic patients with and without PVT, and also to estimate their pathogenetic role in the occurrence of thrombotic events in these patients. Mangia et al. ${ }^{18}$ recently concluded that in cirrhotic patients prothrombotic mutations by themselves are not causative of PVT, suggesting that PVT in these patients is associated with Child-Pugh classes B and C, signs of liver decompensation, large varices with red markings, sclerotherapy, and abdominal surgery. In contradiction to these suggestions, none of our cirrhotic PVT patients demonstrated severe liver dysfunction or had been operated on; only three of them had documented small esophageal varices with no history of variceal bleeding or sclerotherapy; and all patients' serum levels of albumin and fibrinogen, which represent hepatic protein synthesis, showed no correlation with the levels of these antithrombotic proteins.

Family studies would be definitively important in identifying the mutant genes but are impractical, and usually compromised by the denial of the family to be subjected to such an investigation, as happened in our trial. We fol-

Table 2. Abdominal vessel thrombosis and thrombophilia (patients/\%).

\begin{tabular}{|c|c|c|c|c|c|c|c|c|c|c|c|c|}
\hline FVL & FIII ${ }^{\mathrm{G} 20210 \mathrm{~A}}$ & MTHFR $^{\text {C677T }}$ & PRC & PRS & AT & APC-R & LA & MPD & LC & Hcy & PNH & MGN \\
\hline $\begin{array}{c}5 / 29 \\
(17.2 \%)\end{array}$ & $\begin{array}{l}0 / 29 \\
(0 \%)\end{array}$ & $\begin{array}{c}5 / 29 \\
(17.2 \%)\end{array}$ & $\begin{array}{c}5 / 29 \\
(17.2 \%)\end{array}$ & $\begin{array}{c}7 / 29 \\
(24.1 \%)\end{array}$ & $\begin{array}{c}4 / 29 \\
(13.8 \%)\end{array}$ & $\begin{array}{c}1 / 29 \\
(3.4 \%)\end{array}$ & $\begin{array}{l}0 / 29 \\
(0 \%)\end{array}$ & $\begin{array}{c}5 / 29 \\
(17.2 \%)\end{array}$ & $\begin{array}{c}6 / 29 \\
(20.7 \%)\end{array}$ & $\begin{array}{c}5 / 29 \\
(17.2 \%)\end{array}$ & $\begin{array}{l}0 / 29 \\
(0 \%)\end{array}$ & $\begin{array}{c}1 / 29 \\
(3.4 \%)\end{array}$ \\
\hline
\end{tabular}

FVL, Factor V Leiden; FII G20210A, prothrombin G20210A; MTHFRC677T, methylenetetrahydrofolate reductase C677T; PRC, protein C; PRS, protein S; AT deficiencies, antithrombin; APC-R, activated PC resistance; LA, lupus anticoagulant; MPD, myeloproliferative diseases; LC, liver cirrhosis; Hcy, hyperhomocysteinemia; PNH, paroxysmal nocturnal haemospherinouria; MGN, malignancy. 
lowed the convenient screening method for the recognition of these natural anticoagulant deficiencies in patients with liver disease, which was proposed by Pabinger et al. ${ }^{19}$ and initially used by Janssen et al. ${ }^{10}$ These investigators have suggested a formula that is the ratio of PRS or PRC or AT to (factor II+factor $\mathrm{X}) / 2$. If the result is less than $70 \%$, it may signify a deficiency of the tested protein considerably disproportionate to the decreased protein synthesis by the liver. This ratio was $\leq 70 \%$ in all four cirrhotic patients with PCR, PRS, and AT deficiencies. Although these findings favor the genetic origin of these natural inhibitors of coagulation's deficits in our patients, we probably should keep some reservations as this screening formula has not been fully validated yet. Moreover, in patients with venous thrombosis, the thrombotic process may by itself deplete PRC, PRS, and AT. To obviate this, we tested the patients at least one year after the thrombotic event. In all PVT patients who had underlying congenital thrombophilic conditions, the specimens tested confirmed the initial findings.

\section{Mesenteric vein thrombosis}

Five (17.2\%) patients presented with MVT, an uncommon but potentially fatal form of mesenteric ischemia. Four of them had the FVL mutation (three heterozygous and one homozygous) and in one patient no underlying condition was detected. In one of the patients with heterozygous FVL mutation, a combination of PRC, PRS, and AT deficiencies, and APC-R was uncovered in addition. This patient presented with MVT as his first thromboembolic event at the age of 55 years, although thrombosis does occur at a younger age in patients with a coexistence of such multiple thombophilic conditions. ${ }^{20}$ Patients who are heterozygous and homozygous for the FVL mutation have a seven- and 80-fold increased risk of venous thrombosis, respectively, and the strong association of this defect, as well as APC-R, with MVT have been revealed in recent series. ${ }^{21,22}$

\section{Splenic vein thrombosis}

Four (13.8\%) patients presented with SVT. One male patient with no other identifiable underlying thrombophilic risk factor had been recently operated on and a malignant suprasellular brain glioma had been removed partially. Three female patients aged 18, 64, and 53 years old were found to have the MTHFR C677T homozygous mutation associated with increased total homocysteine levels 25.2, 23.8, and $26.4 \mu \mathrm{mol} / \mathrm{L}$, respectively (normal values: 6-15 $\mu \mathrm{mol} / \mathrm{L}$ ), and marginal to severe folate deficiency. About $10 \%$ of the general population are homozygous carriers of this variant (C677T), and in a recent meta-analysis of 53 studies the MTHFR6 77TT genotype was asso- ciated with a $20 \%$ higher risk of venous thrombosis compared with the $677 \mathrm{CC}$ genotype. ${ }^{23}$ Nevertheless, the reports concerning the association of the MTHFR C677T genotype and thrombosis are controversial still, ${ }^{24,25}$ and folate status may play a key role in the appearance of hyperhomocysteinemia in patients with this thermolabile defect. ${ }^{26}$ The coexistence of folate deficiency to a certain extent may be the cause of the increased plasma homocysteine levels in our patients and this might has contributed to the phenotypic expression of the disease. ${ }^{27}$

\section{Hepatic veins thrombosis}

Two (6.9\%) patients presented with HVT (Budd-Chiari syndrome). In one of the patients no underlying risk factor was found and the other one had the homozygous MTHFR C677T mutation, high serum homocysteine levels, and low folate, and was suffering from ET. The etiology of HVT is quite similar to PVT, and MPDs (overt or latent) are the leading cause in $20-50 \%$ of the cases. ${ }^{4,10}$ Wanless et al. ${ }^{28}$ in an autopsy study, found HVT in $6 \%$ of all patients with a history of MPD. A recent study from Northern India ${ }^{29}$ demonstrated the importance of an interaction between genetic and acquired risk factors in HVT, but in contrast to our findings, FVL was found to be the most common (17\%) inherited risk factor. Our findings are compatible with those of a Chinese study $^{30}$ suggesting that both genetic hemochromatosis and the homozygous C677T mutation in the MTHFR gene are important risk factors for HVT.

\section{Conclusions}

Thrombophilia, either congenital or acquired, has major implications in the abdominal vessels, emphasizing the importance of such defects in the pathogenesis of abdominal vessel thrombosis in adult life. Although our results are not supported sufficiently owing to the limited number of patients involved in this study, we suggest that full thrombophilia investigations should be performed on all patients presenting with thrombosis in the major vessels of abdomen.

\section{References}

1. Bagot CN, Arya R. Virchow and his triad: a question of attribution. Br $\mathrm{J}$ Haematol 2008;143:180-90.

2. Huerta C, Johansson S, Wallander MA, et al. Risk Factors and Short-term Mortality of Venous Thromboembolism Diagnosed in the Primary Care Setting in the United Kingdom. Arch Intern Med 2007;167:935-
43.

3. Bezemer ID, van der Meer FJ, Eikenboom $\mathrm{JC}$, et al. The value of family history as a risk indicator for venous thrombosis. Arch Intern Med 2009;169:610-5.

4. Denninger MH, Chait Y, Casadevall N, et al. Cause of portal or hepatic venous thrombosis in adults: the role of multiple concurrent factors. Hepatology 2000;31: 587-91.

5. Valla D, Casadevall N, Huisse MG, et al. Etiology of portal vein thrombosis in adults: A prospective evaluation of primary myeloproliferative disorders. Gastroenterology 1988;94:1063-9.

6. Valla DC, Condat B. Portal vein thrombosis in adults: pathophysiology, pathogenesis and management. J Hepatol 2000;32:86571 .

7. De Stefano V, Teofili L, Leone G, et al. Spontaneous erythroid colony formation as the clue to an underlying myeloproliferative disorder in patients with BuddChiari syndrome or portal vein thrombosis. Semin Thromb Hemost 1997;23:411-8.

8. Elhajj II, Salem ZM, Birjawi GA, et al. Heterozygous prothrombin 20210G/A mutation, associated with hyperhomocysteinemia, and homozygous methylenetetrahydrofolate reductase $677 \mathrm{C} / \mathrm{T}$ mutation, in a patient with portal and mesenteric venous thrombosis. Hematol J 2004;5:5402.

9. Rees DC, Cox M, Clegg JB. World distribution of factor V Leiden. Lancet 1995; 346: 1133-4.

10. Janssen HL, Meinardi JR, Vleggaar FP, et al. Factor V Leiden mutation, prothrombin gene mutation, and deficiencies in coagulation inhibitors associated with BuddChiari syndrome and portal vein thrombosis: results of a case-control study. Blood 2000;96:2364-8.

11. Levoir D, Aubertin JM, Alhenc-Gelas M, et al. Une nouvelle cause hereditaire de thrombose portale: la resistance anormale a la proteine $\mathrm{C}$ activee par mutation Arg506=Gln du gene du facteur V. Gastroenterol Clin Biol 1995;19:729-31.

12. Erkan 0, Bozdayi AM, Disibeyaz S, et al. Thrombophilic gene mutations in cirrhotic patients with portal vein thrombosis. Eur J Gastroenterol Hepatol 2005;17:339-43.

13. Amitrano L, Brancaccio V, Guardascione MA, et al. Inherited coagulation disorders in cirrhotic patients with portal vein thrombosis. Hepatology 2000;31:345-8.

14. Chamouard P, Pencreach E, Maloisel F, et al. Frequent factor II G20210A mutation in idiopathic portal vein thrombosis. Gastroenterology 1999;116:144-8.

15. Primignani M, Martinelli I, Bucciarelli P, et al. Risk factors for thrombophilia in extrahepatic portal vein obstruction. Hepatol- 
ogy 2005;41:603-8.

16. Mohanty D, Shetty S, Ghosh K, et al. Hereditary thrombophilia as a cause of Budd-Chiari syndrome: a study from Western India. Hepatology 2001;34:666-70.

17. Randi ML, Fabris F, Tison T, et al. Prevalence of specific thrombotic accidents in patients with thrombocytosis. Haematologica 1993;25:149-52.

18. Mangia A, Villani MR, Cappucci G, et al. Causes of portal venous thrombosis in cirrhotic patients: the role of genetic and acquired factors. Eur J Gastroenterol Hepatol 2005;17:745-51.

19. Pabinger I, Allaart CF, Hermans J, et al. Hereditary protein C-deficiency: laboratory values in transmitters and guidelines for the diagnostic procedure. Report on a study of the SSC Subcommittee on Protein $\mathrm{C}$ and Protein S. Protein C Transmitter Study Group. Thromb Haemost 1992;68: 470-4.

20. Hertzberg MS, Underwood T, Favaloro EJ. Mesenteric Vein Thrombosis Secondary to Combined Protein C Deficiency and Double Heterozygosity for Factor V Leiden and Prothrombin G20210A. Am J Hematol 1999;62:199-200.

21. Bergenfeldt M, Svensson PJ, Borgstrom A. Mesenteric vein thrombosis due to factor $\mathrm{V}$ Leiden gene mutation Br J Surg 1999; 86: 1059-62.

22. Heresbach D, Pagenault M, Gueret P, et al. Leiden factor $\mathrm{V}$ mutation in four patients with small bowel infarctions. Gastroenterology 1997;113:322-5.

23. Den Heijer M, Lewington S, Clarke R. Homocysteine, MTHFR and risk of venous thrombosis: a meta-analysis of published epidemiological studies. J Thromb Haemost 2005;3:292-9.

24. Salomon 0, Steinberg DM, Zivelin A, et al. Single and Combined Prothrombotic Factors in Patients With Idiopathic Venous Thromboembolism. Arterioscler Thromb Vasc Biol 1999;19:511-8.

25. Kluijtmans LAJ, den Heijer M, Reitsma PH, et al. Thermolabile methylenetetrahydrofolate reductase and factor $\mathrm{V}$ Leiden in the risk of deep vein thrombosis. Thromb Haemost 1998;79:254-8.

26. Kang S, Zhou J, Wong PW, et al.
Intermediate homocysteinemia: a thermolabile variant of methylenetetrahydrofolate reductase. Am J Hum Genet 1988;43:41421.

27. Starakis I, Mougiou A, Leonidou L, et al. Splenic thrombosis in three patients with moderate hyperhomocysteinemia, low folate and the C677T variant of the methylenetetrahydrofolate reductase (MTHFR) gene. Thromb Haemost 2005;94:1333-4.

28. Wanless IR, Peterson P, Das A, et al. Hepatic vascular disease and portal hypertension in polycythemia vera and agnogenic myeloid metaplasia: a clinicopathological study of 145 patients examined at autopsy. Hepatology 1990;12:1166-74.

29. Bhattacharyya M, Makharia G, Kannan M, et al. Inherited prothrombotic defects in Budd-Chiari syndrome and portal vein thrombosis: a study from North India. Am J Clin Pathol 2004;121:844-7.

30. Li XM, Wei YF, Hao HL, et al. Hyperhomocysteinemia and the MTHFR C677T mutation in Budd-Chiari syndrome. Am J Hematol 2002;71:11-4. 